\title{
10-Year Fracture Risk in Postmenopausal Women with Osteopenia and Osteoporosis in South Korea
}

\author{
Yeon-Hee Baek ${ }^{1,2, *}$, Sun Wook Cho ${ }^{3, *}$, Han Eol Jeong ${ }^{1}$, Ju Hwan Kim ${ }^{1}$, Yunji Hwang ${ }^{2,4}$, Jeffrey L. Lange ${ }^{4}$, \\ Ju-Young Shin ${ }^{1,5,6}$
}

${ }^{1}$ School of Pharmacy, Sungkyunkwan University, Suwon; ${ }^{2}$ Amgen Korea; ${ }^{3}$ Department of Internal Medicine, Seoul National University Hospital, Seoul National University College of Medicine, Seoul, Korea; ${ }^{4}$ Center for Observational Research, Amgen, Thousand Oaks, CA, USA; ${ }^{5}$ Department of Biohealth Regulatory Science, Sungkyunkwan University, Suwon; ${ }^{6}$ Department of Clinical Research Design \& Evaluation, Samsung Advanced Institute for Health Sciences \& Technology, Sungkyunkwan University, Seoul, Korea

Background: In South Korea, women aged 66 years are eligible for complimentary bone mineral density (BMD) screening via the National Screening Program for Transitional Ages. We aimed to evaluate the 10-year fracture risk in women receiving BMD screening between January 2008 and December 2015.

Methods: BMD was classified as normal (T-score $\geq-1.0$ standard deviation [SD]), osteopenia (T-score $<-1.0 \mathrm{SD}$ and $>-2.5 \mathrm{SD}$ ), and osteoporosis (T score $\leq-2.5 \mathrm{SD}$ ) from dual-energy X-ray absorptiometry. Follow-up continued from the screening date until a diagnosis for clinical fragility fracture (including sites of the vertebrae, hip, pelvis, clavicle, humerus, forearm, wrist, lower leg, and ankle), censored at the earliest date of trauma, death, or December 2017; fracture was ascertained using diagnostic codes from the National Health Insurance Service database. A multivariable Cox proportional hazard model was used to estimate hazard ratios (HRs) and 95\% confidence intervals (CIs) for the risk of fracture in women with osteopenia or osteoporosis relative to women with normal BMD.

Results: Among the 271,197 women screened, $44.0 \%$ had osteopenia and $35.2 \%$ had osteoporosis. The 10 year cumulative incidence of fragility fractures was $31.1 \%, 37.5 \%$, and $44.3 \%$ in women with normal BMD, osteopenia, and osteoporosis, respectively. Fracture risk was higher in women with osteopenia (HR, 1.31; 95\% CI, 1.28 to 1.34 ) and osteoporosis (HR, 1.68; $95 \%$ CI, 1.64 to 1.72) than in women with normal BMD.

Conclusion: Women with osteopenia and women with osteoporosis, identified by the national BMD screening program, demonstrated a substantially elevated risk of fracture.

Keywordls: Bone density; Bone diseases, metabolic; Fractures, bone; Osteoporosis; Postmenopause

\section{INTRODUCTION}

Osteoporosis is a metabolic bone disease characterized by loss

Received: 28 July 2021, Revised: 15 September 2021,

Accepted: 6 October 2021

Corresponding author: Ju-Young Shin

School of Pharmacy, Sungkyunkwan University, 2066 Seobu-ro, Jangan-gu, Suwon 16419, Korea

Tel: +82-31-290-7702, Fax: +82-31-299-4377, E-mail: shin.jy@skku.edu of bone mass and deteriorated bone microstructure, with a consequent increase in bone fragility and long-term fracture risk [1]. Impaired bone turnover cycles due to estrogen deficiency are

\section{Copyright $(\odot 2021$ Korean Endocrine Society}

This is an Open Access article distributed under the terms of the Creative Commons Attribution Non-Commercial License (https://creativecommons.org/ licenses/by-nc/4.0/) which permits unrestricted non-commercial use, distribution, and reproduction in any medium, provided the original work is properly cited.

*These authors contributed equally to this work. 
associated with low bone mineral density (BMD) in postmenopausal women [2], resulting in an increased risk of fracture at sites such as the vertebrae and hip [3-5]. Osteopenia has traditionally been considered a precursor of future osteoporosis rather than an abnormal bone state with inherent fracture risk. However, the incidence of fragility fractures in osteopenia is substantial; $82 \%$ of postmenopausal women with fragility fractures have osteopenia or normal BMD [6] in the United States. Osteoporosis treatments are generally not indicated for fracture prevention in patients with osteopenia based on the current clinical recommendations; thus, a considerable number of women are at risk of fracture.

The prevalence of osteopenia ranges from $27.1 \%$ to $51.6 \%$, with a reportedly high prevalence among postmenopausal women in Asian countries, where the population is aging rapidly [79]. Meanwhile, there is a demand for ethnic-specific evidence owing to the high heterogeneity in BMD distributions and differential fracture risks across ethnicities [10]. Asian postmenopausal women have the lowest BMD among five different ethnic groups (Asian vs. Caucasian [mean T-score]: -1.22 vs. -0.89 ) [10]. A longitudinal study from Hong Kong demonstrated that lower BMD was associated with a 1.5-fold and 1.7-fold increased risk of fragility fractures per standard deviation (SD) reduction in BMD at the spine and hip, respectively [11]. However, owing to the limitation of convenience sampling, evidence gaps remain in the assessment of long-term fracture risk in postmenopausal women with osteopenia in the East Asian population.

In South Korea, all women aged 66 years are eligible for BMD analysis through the National Health Screening Program, and their clinical outcomes can be followed for 10 years by retrieving records from the National Health Insurance Database via data linkage. Therefore, we sought to identify the long-term fracture risk over 10 years using BMD measured in a population based screening program.

\section{METHODS}

\section{Data source}

In South Korea, the National Health Insurance Service (NHIS), a single-payer healthcare system covering the entire population, reimburses costs based on billing records from health care providers. The NHIS launched a national general health screening program for chronic diseases in 1995 [12], followed by the introduction of the National Screening Program for Transitional Ages (NSPTA) in 2007 [13]. The NSPTA examines individuals aged 40 and 66 years, and a complimentary BMD screening is provided to women aged 66 years as part of the NSPTA [13]. The NHIS informs subjects of their eligibility for the NSPTA screening by sending notification and reminders via post; participants can select sites among the listed healthcare centers or hospitals. Among the individuals who were eligible for the NSPTA health examination, 77\% underwent examination in 2015 [14]. Using these data, the NHIS created a database of claims information that encompassed diagnosis records, procedures, surgery, prescriptions, or health screening records.

Furthermore, the NHIS created a database that covered demographic information (age, sex, and income level), national health screening data, and health utilization data (inpatient and outpatient visits, including prescriptions, procedures, and diagnosis records) [15]. The database also contains information on completely enumerated death records obtained from the vital statistics of South Korea. Diagnosis records were coded using the International Classification of Diseases, Tenth Revision (ICD-10). The overall positive predictive value of the diagnoses in the South Korean health insurance claims database was $82 \%$ [16]. In the database, each individual has an anonymized unique personal identification number, which enabled individual-level follow-up for 10 years via individual-level linkage across databases (National Health Screening Database, Health Care Utilization Database, and Vital Statistics of Statistics Korea).

The NHIS extracted and provided 50\% randomly sampled data from approximately 1 million women aged 66 years who underwent the NSPTA health examination and BMD screening between January 1, 2008, and December 31, 2015. Due to the NHIS's data access policy, a maximum of $50 \%$ of randomly selected sample data was available for external researchers due to privacy issues. From the nationwide healthcare claims database, all patients' reimbursed healthcare encounters (including diagnostic records, prescriptions, or procedures) were retrieved, and therefore, longitudinal patient-level follow-up was possible in our study.

\section{Study subjects}

We conducted a retrospective cohort study over 10 years, using data from postmenopausal women who underwent BMD screening tests in the NSPTA health examination at the age of 66 years between January 1, 2008, and December 31, 2015. Subjects were followed up until December 31, 2017, to ensure at least 2 years of follow-up.

\section{Exposure}

BMD was measured using dual-energy X-ray absorptiometry 
(DXA) [17] primarily at the spine; if this was impossible (due to vertebral fracture or surgery), BMD was measured at the femoral neck [18]. The subjects were categorized into normal BMD (T score $\geq-1.0$ ), osteopenia (T-score $<-1.0 \mathrm{SD}$ and $>-2.5 \mathrm{SD}$ ), and osteoporosis ( $\mathrm{T}$ score $\leq-2.5 \mathrm{SD}$ ), according to the World Health Organization (WHO) criteria [19].

Cohort entry was defined as the date of BMD screening. A minimum of 1 year of database history prior to cohort entry was required for all subjects to ascertain study eligibility and to assess baseline demographic and clinical characteristics. The following subjects were excluded (Supplemental Table S1): (1) subjects with a history of cancer, Paget's disease, human immunodeficiency virus (HIV) infection, or osteogenesis imperfecta to exclude the impact of residual confounding from conditions known to be associated with an increased risk of fracture; (2) subjects with a history of fracture or trauma, as these are major and time-dependent risk factors for subsequent fragility fractures; (3) subjects who had chronic exposure to glucocorticoids ( $\geq 5 \mathrm{mg}$ prednisolone-equivalent steroid/day for $\geq 3$ months) to exclude glucocorticoid-induced osteoporosis fracture risk [20]; and (4) subjects who previously received osteoporosis treatment.

This study was approved by the Institutional Review Board of Sungkyunkwan University (approval number: SKKU 201902-006). Written informed consent was not applicable, as this study used anonymized subject data.

\section{Outcome}

Subjects with an incident fracture event of the vertebrae, hip, or non-hip and non-vertebral (NHNV) sites, as defined in a previous publication by Lee et al. [21], during the follow-up period were considered to develop an outcome (Supplemental Table $\mathrm{S} 1$ ). To ascertain cases of fragility fractures, subjects who experienced any traumatic event were censored at the date the event occurred. Subjects were followed from cohort entry until the outcome of interest (fragility fracture), trauma, death, or end of the study period (December 31, 2017), whichever occurred first. We censored the observation of subjects if any censoring event occurred rather than excluding these subjects at the start of the follow-up because excluding these patients would introduce additional selection bias by using future information to determine the inclusion of study subjects. As our analysis was based on a nationwide healthcare claims database, artificial dropouts due to transfer or referral to different health care providers or patient withdrawal were unlikely to occur.

\section{Potential confounders}

We selected potential confounders based on previous literature on the factors associated with fragility fractures [22] and assessed these confounders in the year before cohort entry. Potential confounders included income level, lifestyle (smoking and alcohol consumption frequency), body mass index (BMI; categorized into underweight $\left[<18.5 \mathrm{~kg} / \mathrm{m}^{2}\right]$, normal $\left[18.5-22.9 \mathrm{~kg} / \mathrm{m}^{2}\right]$, overweight [23.0-24.9 kg/m²], obese I [25.0-29.9 kg/m $\left.{ }^{2}\right]$, or obese II $\left[\geq 30.0 \mathrm{~kg} / \mathrm{m}^{2}\right]$ according to the Asian criteria of the WHO) [23], baseline clinical conditions, including risk factors for fracture, such as subclinical hyperthyroidism [24], diabetes mellitus [25], hypertension [26], chronic obstructive pulmonary disease (COPD)/asthma [27,28], and rheumatoid arthritis [29] (listed in Supplemental Table S1), and comedications (calcium and vitamin D, anticonvulsants, proton pump inhibitors, thyroid hormones, selective serotonin reuptake inhibitors, and thiazolidinediones). We also assessed the use of osteoporosis medications during the patient follow-up (including oral bisphosphonates [alendronate, risedronate, and ibandronate, with or without vitamin D derivatives], intravenous bisphosphonates [ibandronate, pamidronate, and zoledronate], and selective estrogen receptor modulators [SERMs; raloxifene and bazedoxifene]). Clinical diagnoses were determined and coded according to the ICD-10 by clinicians based on relevant clinical guidelines and clinical judgment. We additionally retrieved data on the conduct of BMD tests within 2 years after cohort entry to assess whether the frequency of subsequent BMD tests was different among the three BMD groups.

\section{Statistical analysis}

The demographic and clinical characteristics of the study subjects with normal BMD, osteopenia, or osteoporosis were described. The 10-year cumulative incidence was defined as the number of incident fracture events during the study period divided by the number of persons at risk within the cohort. It represented the proportion of women with new fractures among women at risk over 10 years. Cumulative incidence curves were derived using the Kaplan-Meier approach, presenting the time to incident fracture for all three groups during follow-up. Significance was determined using a log-rank test. We constructed Cox proportional hazard models to estimate hazard ratios (HRs) and their 95\% confidence intervals (CIs) for the risk of fracture in subjects with osteopenia or osteoporosis versus those with normal BMD. The outcome models were adjusted for income, smoking, alcohol consumption, BMI, comorbidities (thyroid dysfunction, asthma, COPD, rheumatoid arthritis, hypertension, 
myocardial infarction, heart failure, diabetes mellitus, dyslipidemia, stroke, chronic kidney disease, and gastrointestinal disorders) and comedications (thyroid hormones, calcium and vitamin D, anticonvulsants, proton pump inhibitors, selective serotonin reuptake inhibitors, and benzodiazepines). Further analyses were performed to assess the outcomes of each fracture type.

To describe the range of fracture risk among those receiving an osteoporosis treatment or not, we conducted stratified analyses by any use of osteoporosis medication between the BMD screening date and end of follow-up (fracture occurrence, censoring event, or study end date). Moreover, given bone deterioration over time may be high in post-menopausal women, some women with normal or osteopenic baseline BMD may have progressed to osteoporosis over the 10-year follow-up. Thus, to limit this potential misclassification of differences in baseline and subsequent BMD categories during the follow-up period, the analyses were stratified by any use of osteoporosis medications during the follow-up period to capture the patients most likely to have progression to osteoporosis. All statistical analyses were performed using SAS Enterprise Guide version 7.1 for Windows (SAS Institute Inc., Cary, NC, USA). A two-tailed value of $P<0.05$ indicated significance.

\section{RESULTS}

\section{Baseline characteristics}

Among 335,363 women who underwent DXA BMD testing from January 1, 2008, to December 31, 2015, we included 271,197 women after excluding subjects with a history of osteoporosis medication use $(n=26,658)$, fracture $(n=19,830)$, cancer ( $n=15,662)$, chronic glucocorticoid use $(n=1,087)$, trauma $(n=913)$, HIV infection $(n=12)$, and Paget's disease $(n=4)$. Among 271,197 women who met the eligibility criteria, 56,189 (20.7\%) had normal BMD, 119,458 (44.0\%) had osteopenia, and 95,550 (35.2\%) had osteoporosis, based on their BMD Tscores (Fig. 1). Clinically compared to normal BMD group, osteoporotic patients were less likely to be obese, had generally fewer co-morbidities, and were more likely to receive subsequent BMD test (Table 1).

\section{Fracture risk in different BMD groups}

Over a mean follow-up of 4.54 years, 61,671 incident fracture events occurred and over half of the events occurred in women with normal BMD or osteopenia (15.9\% and $42.5 \%$, respectively), while $41.6 \%$ of the events occurred in women with osteoporosis. The 10-year cumulative incidence of all fractures in postmenopausal women with normal BMD, osteopenia, and osteoporosis was $31.1 \%, 37.5 \%$, and $44.3 \%$, respectively (Fig. 2A). Likewise, the 10-year cumulative incidence of vertebral fracture was higher in women with osteopenia than in women with normal BMD (10.9\% vs. 6.5\%) (Fig. 2B), as was the cumulative incidence of hip fracture (2.2\% vs. 1.9\%) (Fig. 2C) and NHNV fracture (30.7\% vs. $26.5 \%$ ) (Fig. 2D). The cumulative incidence of fracture among women with osteoporosis was largely com-

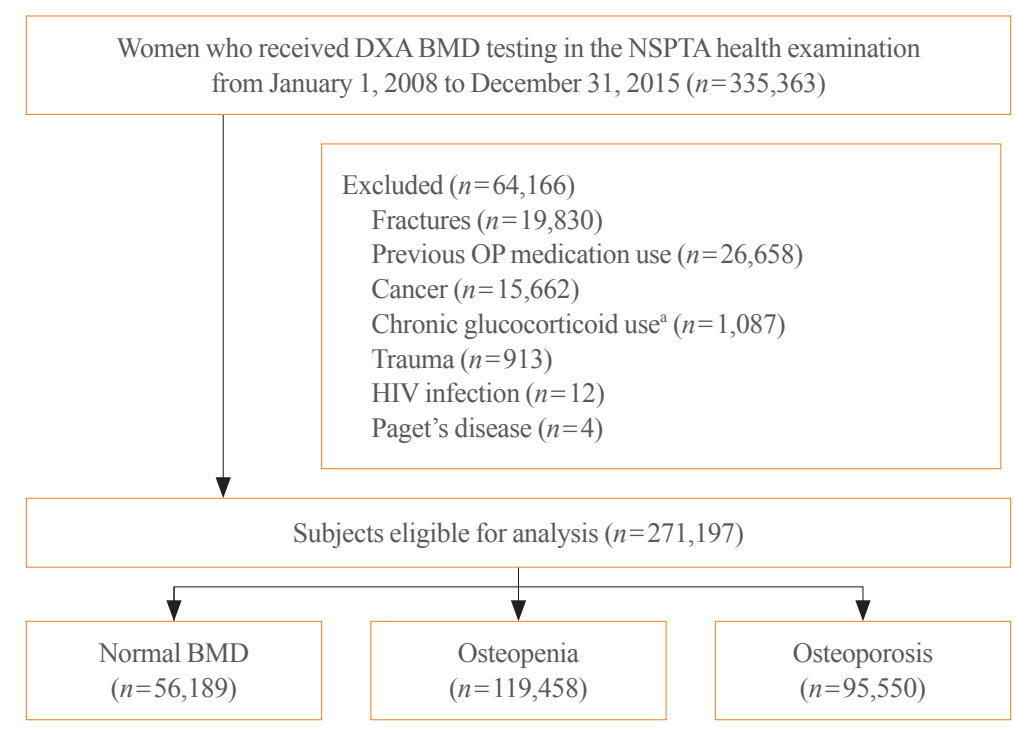

Fig. 1. Selection of study subjects who underwent dual-energy X-ray absorptiometry (DXA) bone mineral density (BMD) screening from the National Screening Program for Transitional Ages (NSPTA). OP, osteoporosis; HIV, human immunodeficiency virus. ${ }^{a} \geq 5$ mg prednisolone-equivalent steroid/day for $\geq 3$ months. 


\begin{tabular}{|c|c|c|c|}
\hline Characteristic $^{\mathrm{a}}$ & Normal BMD $(n=56,189)$ & Osteopenia $(n=119,458)$ & Osteoporosis $(n=95,550)$ \\
\hline \multicolumn{4}{|l|}{ BMI } \\
\hline Underweight $\left(<18.5 \mathrm{~kg} / \mathrm{m}^{2}\right)$ & $280(0.5)$ & $1,149(1.0)$ & $2,855(3.0)$ \\
\hline Normal $\left(18.5-22.9 \mathrm{~kg} / \mathrm{m}^{2}\right)$ & $11,679(20.8)$ & $32,876(27.5)$ & $35,604(37.3)$ \\
\hline Overweight (23.0-24.9 kg/m²) & $14,389(25.6)$ & $32,818(27.5)$ & $25,639(26.8)$ \\
\hline Obese I $\left(25.0-29.9 \mathrm{~kg} / \mathrm{m}^{2}\right)$ & $24,998(44.5)$ & $46,326(38.8)$ & $28,487(29.8)$ \\
\hline Obese II $\left(\geq 30.0 \mathrm{~kg} / \mathrm{m}^{2}\right)$ & 4,833 (8.6) & $6,268(5.3)$ & $2,942(3.1)$ \\
\hline \multicolumn{4}{|l|}{ Smoking } \\
\hline Never & $49,625(88.3)$ & $104,882(87.8)$ & $82,902(86.8)$ \\
\hline Past but stopped & $616(1.1)$ & $1,191(1.0)$ & $802(0.8)$ \\
\hline Current & $847(1.5)$ & $1,846(1.6)$ & $1,929(2.0)$ \\
\hline Alcohol consumption ( $\geq 1$ time per week) & $4,477(8.0)$ & $8,980(7.5)$ & $6,421(6.7)$ \\
\hline \multicolumn{4}{|l|}{ Baseline clinical condition } \\
\hline Hypothyroidism & $3,419(6.1)$ & $6,380(5.3)$ & $4,330(4.5)$ \\
\hline Hyperthyroidism & $1,140(2.0)$ & $2,465(2.1)$ & $1,786(1.9)$ \\
\hline Other thyroid disorder & $4,934(8.8)$ & $9,808(8.2)$ & $6,831(7.2)$ \\
\hline Asthma & $8,014(14.3)$ & $16,647(13.9)$ & $12,517(13.1)$ \\
\hline COPD & $10,430(18.6)$ & $21,401(17.9)$ & $15,300(16.0)$ \\
\hline Rheumatoid arthritis & $2,913(5.2)$ & $5,709(4.8)$ & $3,872(4.1)$ \\
\hline Hypertension & $30,919(55.0)$ & $58,918(49.3)$ & $40,708(42.6)$ \\
\hline Diabetes mellitus & $15,226(27.1)$ & $27,597(23.1)$ & $17,893(18.7)$ \\
\hline Dyslipidemia & $25,330(45.1)$ & $49,222(41.2)$ & $32,760(34.3)$ \\
\hline Stroke & $3,682(6.55)$ & $7,624(6.38)$ & $5,744(6.0)$ \\
\hline Chronic kidney disease & $348(0.6)$ & $700(0.6)$ & $513(0.5)$ \\
\hline \multicolumn{4}{|l|}{ Previous medication use } \\
\hline Thyroid hormones & $2,295(4.1)$ & $4,259(3.6)$ & $2,759(2.9)$ \\
\hline Calcium and vitamin D & $4,227(7.5)$ & $12,796(10.7)$ & $9,914(10.4)$ \\
\hline \multicolumn{4}{|c|}{ Subsequent BMD test within 2 years after cohort entry } \\
\hline Yes & $15,081(26.8)$ & $37,104(31.1)$ & $40,860(42.8)$ \\
\hline
\end{tabular}

Values are expressed as number (\%).

BMD, bone mineral density; DXA, dual-energy X-ray absorptiometry; BMI, body mass index; COPD, chronic obstructive pulmonary disease.

${ }^{a}$ All variables were nominally statistically different $(P<0.01)$ among the three cohorts with exception to chronic kidney disease.

parable to that among women with osteopenia. The risk of fragility fractures was higher in women with osteopenia than in women with normal BMD (HR, 1.31; 95\% CI, 1.28 to 1.34) (Table 2). Similar results were observed for women with osteoporosis when compared with women with normal BMD (HR, $1.68 ; 95 \%$ CI, 1.64 to 1.72 ).

Table 3 shows the risk of overall fracture stratified by any use of osteoporosis treatment during the follow-up. Consistent with the major findings, the association between baseline BMD group and fracture risk remained consistent where the incidence was the highest among osteoporosis, followed by osteopenia and normal BMD group in both treated and non-treated groups. Among women who initiated an osteoporosis treatment during follow-up, overall fracture incidence rates per 100 person-years (PY) were 2.92, 3.41, and 4.88 in women with normal BMD, osteopenia, and osteoporosis, respectively. On the other hand, the overall fracture incidence rates per $100 \mathrm{PY}$ among those who did not receive osteoporosis treatment during follow-up were 3.92, 5.50, and 8.09 in women with normal BMD, osteopenia, and osteoporosis, respectively. Among women diagnosed with osteoporosis at the index screening, 59.8\% initiated an osteoporosis treatment during follow-up and their 10-year cumu- 
Overall fracture

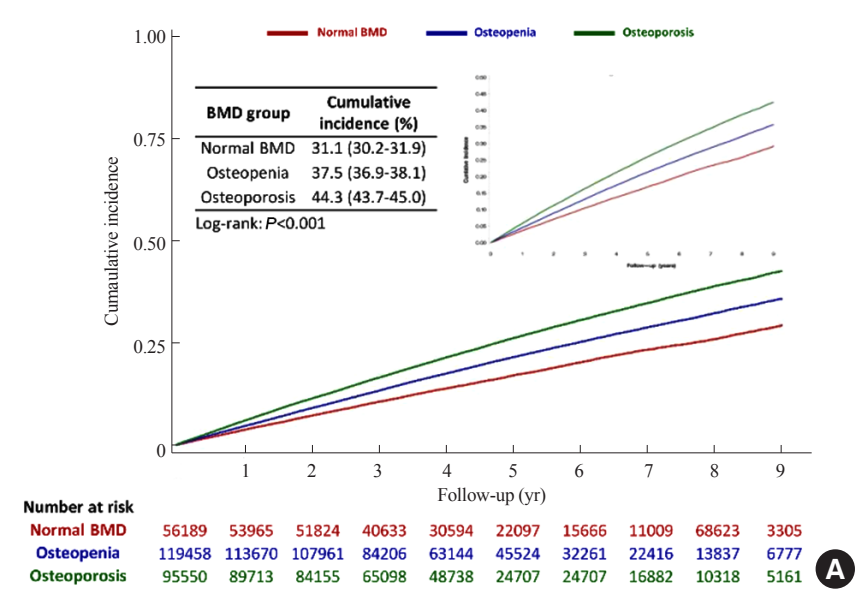

Vertebral fracture

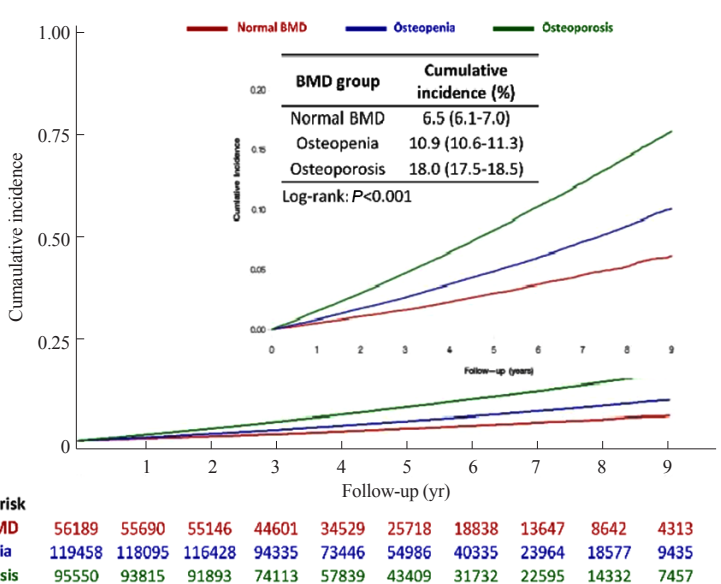

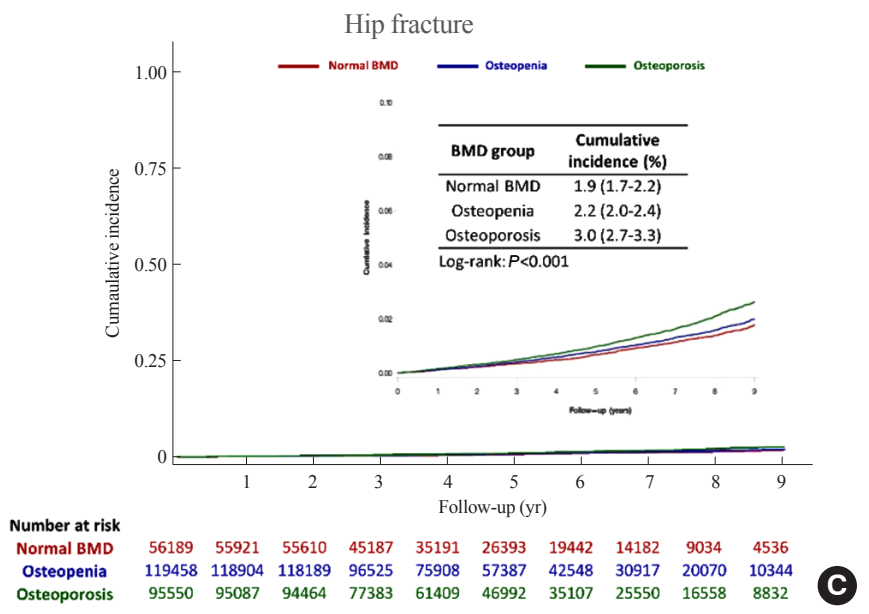

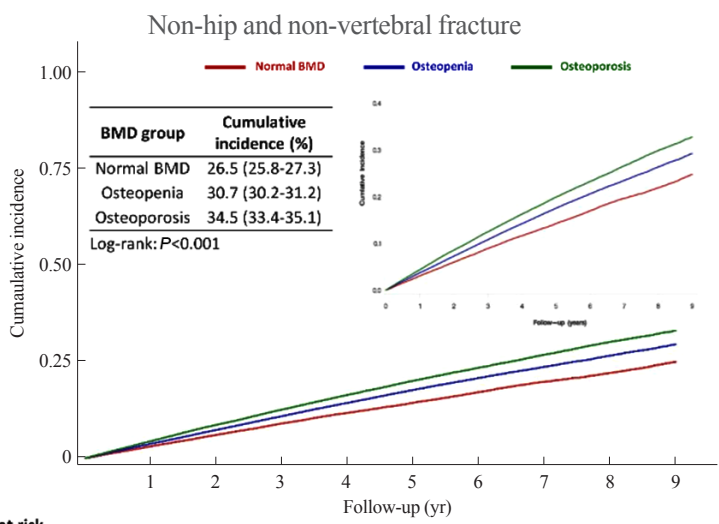

Number at risk

$\begin{array}{lllllllllll}\text { Normal BMD } & 56189 & 54271 & 52416 & 41345 & 31338 & 22811 & 16283 & 11521 & 7173 & 3496\end{array}$ $\begin{array}{lcccccccccc}\text { Osteopenia } & 119458 & 114590 & 109871 & 86506 & 65574 & 47788 & 34251 & 24124 & 15089 & 7506 \\ \text { Osteoporosis } & 95550 & 91064 & 86655 & 68102 & 51841 & 38069 & 27382 & 19106 & 11930 & 6110\end{array}$

Fig. 2. Cumulative incidence curves for the time to (A) overall, (B) vertebral, (C) hip, and (D) non-hip and non-vertebral fractures after dualenergy X-ray absorptiometry (DXA) bone mineral density (BMD) screening test in subjects with normal BMD, osteopenia, or osteoporosis.

lative incidence was $38.3 \%$; $41.2 \%$ did not initiate an osteoporosis treatment during follow-up and their 10-year cumulative incidence was $48.9 \%$. While similar trends were observed in the analyses stratified by fracture subtypes (Supplemental Tables S2-S4), the incidence of hip fracture did not differ between normal and osteopenia group among the treated subgroup. The 10year cumulative incidence of fracture among women with normal and osteopenia baseline BMD who did not receive osteoporosis treatment was $29.4 \%$ and $37.8 \%$, respectively. Censoring frequency due to the occurrence of trauma or death was largely similar across the BMD groups (Supplemental Table S5).

\section{DISCUSSION}

We conducted a large population-based cohort study to analyze postmenopausal fracture risk in South Korea. Long-term frac- ture risk was substantial among 271,197 postmenopausal women in South Korea. Following the national screening, the 10year cumulative fracture incidence in women with normal BMD was $31.1 \%$, while $37.5 \%$ and $44.3 \%$ in women with osteopenia and osteoporosis, respectively. Although the fracture rate was the highest in women with osteoporosis, over half of the fracture events that occurred throughout the 10-year follow-up period were in women with osteopenia or normal BMD. Postmenopausal women with osteopenia had a 1.31-fold increased risk of overall fracture compared with women with normal BMD, independent of other risk factors, including medical history and comedication use. The national BMD screening program in South Korea provided useful insights into identifying postmenopausal women at an increased risk of fracture.

Consistent with our findings, where most fractures occurred in postmenopausal women with modest BMD (T-score $>-2.5$ 
Table 2. Risk of Fracture in Subjects with Osteopenia or Osteoporosis versus Subjects with Normal BMD

\begin{tabular}{|c|c|c|c|c|c|c|}
\hline Fracture site & No. of subjects & No. of events & $\begin{array}{l}\text { PY } \\
\text { at risk }\end{array}$ & $\begin{array}{c}\text { Incidence rate per } \\
100 \mathrm{PY} \\
(95 \% \mathrm{CI})^{\mathrm{a}}\end{array}$ & $\begin{array}{l}\text { Unadjusted HR } \\
\qquad(95 \% \mathrm{CI})\end{array}$ & $\begin{array}{c}\text { Adjusted HR } \\
(95 \% \mathrm{CI})^{\mathrm{b}}\end{array}$ \\
\hline
\end{tabular}

\section{Overall fracture}

Normal BMD

Osteopenia

56,189

9,804

262,170

$3.74(3.67-3.81)$

$\operatorname{Ref}(1.00)$

$\operatorname{Ref}(1.00)$

Osteoporosis

119,458

26,223

545,481

$4.81(4.75-4.86)$

$1.29(1.26-1.32)$

$1.31(1.28-1.34)$

Vertebral fracture

Normal BMD

95,550

25,644

424,087

$6.05(5.98-6.12)$

$1.62(1.58-1.66)$

$1.68(1.64-1.72)$

$\begin{array}{lr}\text { Normal BMD } & 56,189 \\ \text { Osteopenia } & 119,458 \\ \text { Osteoporosis } & 95,550 \\ \text { Hip fracture } & \end{array}$

$\begin{array}{ll}6,402 & 610,324 \\ 8,746 & 481,328\end{array}$

$0.63(0.6-0.66)$

$\operatorname{Ref}(1.00)$

$\operatorname{Ref}(1.00)$

$1.05(1.02-1.07)$

$1.66(1.57-1.75)$

$1.71(1.62-1.80)$

Hip fracture

Normal BMD

56,189

451

291,800

$1.82(1.78-1.86)$

2.87 (2.73-3.02)

$3.09(2.94-3.25)$

Osteopenia

119,458

1,119

626,618

$0.15(0.14-0.17)$

$\operatorname{Ref}(1.00)$

$\operatorname{Ref}(1.00)$

Osteoporosis

95,550

1,158

505,568

$0.18(0.17-0.19)$

$1.15(1.03-1.28)$

$1.20(1.07-1.34)$

NHNV fracture

Normal BMD

56,189

8,323

266,931

$0.23(0.22-0.24)$

$1.47(1.32-1.64)$

$1.59(1.42-1.78)$

Osteopenia

119,458

21,532

561,106

3.12 (3.05-3.18)

$\operatorname{Ref}(1.00)$

$\operatorname{Ref}(1.00)$

Osteoporosis

95,550

19,716

444,466

3.84 (3.79-3.89)

$1.23(1.20-1.26)$

$1.24(1.21-1.27)$

$4.44(4.38-4.5)$

$1.42(1.39-1.46)$

$1.46(1.42-1.50)$

PY, person-years; CI, confidence interval; HR, hazard ratio; BMD, bone mineral density; NHNV, non-hip and non-vertebral.

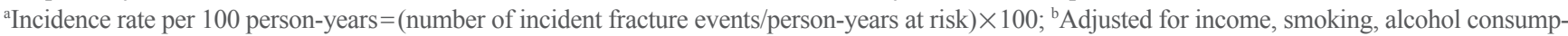
tion, body mass index, comorbidities (thyroid dysfunction, asthma, chronic obstructive pulmonary disease, rheumatoid arthritis, hypertension, myocardial infarction, heart failure, diabetes mellitus, dyslipidemia, stroke, chronic kidney disease, gastrointestinal disorders) and comedications (thyroid hormones, calcium and vitamin D, anticonvulsants, proton pump inhibitors, selective serotonin reuptake inhibitors, and benzodiazepines).

Table 3. Risk of Overall Fracture Stratified by the Use of Osteoporosis Treatment during the Follow-up in Subjects with Osteopenia or Osteoporosis versus Subjects with Normal BMD

\begin{tabular}{|c|c|c|c|c|c|c|c|}
\hline Stratification & $\begin{array}{c}\text { No. of } \\
\text { subjects }\end{array}$ & $\begin{array}{l}\text { No. of } \\
\text { events }\end{array}$ & $\begin{array}{l}\mathrm{PY} \\
\text { at risk }\end{array}$ & $\begin{array}{c}\text { 10-Year } \\
\text { cumulative } \\
\text { incidence, } \%\end{array}$ & $\begin{array}{c}\text { Incidence rate per } \\
100 \mathrm{PY} \\
(95 \% \mathrm{CI})^{\mathrm{a}}\end{array}$ & $\begin{array}{l}\text { Crude HR } \\
(95 \% \mathrm{CI})\end{array}$ & $\begin{array}{c}\text { Adjusted HR } \\
(95 \% \mathrm{CI})^{\mathrm{b}}\end{array}$ \\
\hline
\end{tabular}

With any osteoporosis treatment use during follow-up ${ }^{c}$

$\begin{array}{lrrrrrrc}\text { Normal } & 8,067 & 1,359 & 46,574 & 26.5 & 2.92(2.77-3.07) & \operatorname{Ref}(1.00) & \operatorname{Ref}(1.00) \\ \text { Osteopenia } & 33,657 & 6,191 & 181,420 & 30.3 & 3.41(3.33-3.50) & 1.19(1.12-1.26) & 1.21(1.14-1.29) \\ \text { Osteoporosis } & 57,120 & 13,178 & 270,059 & 38.3 & 4.88(4.80-4.96) & 1.75(1.66-1.86) & 1.85(1.74-1.95)\end{array}$

Without any osteoporosis treatment use during follow-up

\begin{tabular}{lrrrrrrr} 
Normal & 48,122 & 8,445 & 215,596 & 29.4 & $3.92(3.84-4.00)$ & $\operatorname{Ref}(1.00)$ & $\operatorname{Ref}(1.00)$ \\
Osteopenia & 85,801 & 20,032 & 364,061 & 37.8 & $5.50(5.43-5.58)$ & $1.40(1.37-1.44)$ & $1.43(1.40-1.47)$ \\
Osteoporosis & 38,430 & 12,466 & 154,028 & 48.9 & $8.09(7.96-8.23)$ & $2.06(2.00-2.12)$ & $2.17(2.11-2.23)$ \\
\hline
\end{tabular}

BMD, bone mineral density; PY, person-years; CI, confidence interval; HR, hazard ratio.

${ }^{a}$ Incidence rate per 100 person-years $=($ number of incident fracture events/person-years at risk $) \times 100$; ${ }^{b}$ Adjusted for income, smoking, alcohol consumption, body mass index, comorbidities (thyroid dysfunction, asthma, chronic obstructive pulmonary disease, rheumatoid arthritis, hypertension, myocardial infarction, heart failure, diabetes mellitus, dyslipidemia, stroke, chronic kidney disease, gastrointestinal disorders) and comedications (thyroid hormones, calcium and vitamin D, anticonvulsants, proton pump inhibitors, selective serotonin reuptake inhibitors, and benzodiazepines); ${ }^{\mathrm{c} O s t e o p o r o s i s}$ treatment status was ascertained between the index BMD screening date and end of follow-up (fracture occurrence, censoring event, or study end date). 
SD), $82 \%$ and $73 \%$ of fractures occurred in women with osteopenia and normal BMD in the United States [6] and Australia [30], respectively. Although differences in demographics across the BMD groups precluded a direct comparison, osteopenia and osteoporosis were more prevalent in our data $(44.0 \%$ and $35.2 \%$, respectively) than in a study of postmenopausal women from the United States (51.4\% and 15.4\%) [31] and Australia (48.0\% and $14.5 \%$ ) [30], most of whom were Caucasian [11]. We observed higher fracture incidences per $100 \mathrm{PY}$ in the normal BMD and osteopenia groups (4.81 and 6.05, respectively) compared with those in Danish women (3.55 and 4.74, respectively) [32].

In the Asian population, a study conducted in Hong Kong predicted the 10-year incidence of fragility fracture risk in women (mean age, 63.4 years) recruited from 1995 to 2002 [11]. The 10-year cumulative incidence in our study was higher than the predicted 10-year absolute risk in women from Hong Kong with three to eight other clinical risk factors (the predicted 10 -year fracture incidence for normal BMD, osteopenia, and osteoporosis in Hong Kong were 13\%, 23.4\%, and 30\%, respectively) [11]. This discrepancy in the 10-year fracture risk between South Korea and Hong Kong may have occurred because of the exclusion of women with a personal/family history of osteoporosis, other bone-related disorders, and premature menopause (age <40 years) in the Hong Kong study, resulting in healthier study participants relative to postmenopausal women in East Asia overall [11,33]. We observed a similar vertebral fracture incidence compared with that in Japanese women (mean age, 65.0 years) from the regional health screening data between 1994 and 1995 (incidence per 100 PY in South Korea vs. Japan: 1.23 vs. 1.24) [34]. Hip fracture incidence per 100 PY was higher or comparable with our results in China, Hong Kong, and Taiwan (0.18 [35], 0.27 [36], and 0.51 [37], respectively). Therefore, our results confirm that postmenopausal fracture risk is still substantial in Asia, despite advances in diagnosis and preventive treatments in recent years.

Global epidemiological data suggest that the burden of fragility fractures remains a major public health issue in Asia owing to the forecasted rapid aging of the population in the upcoming decades [38]. However, fragility fractures have practical opportunities for disease prevention and modification by implementation of population-driven preventive strategies and early identification of at-risk individuals. Even a small increase in bone mass may markedly reduce the burden of fractures at a population level; randomized trials of antiresorptive agents demonstrated a $35 \%$ to $50 \%$ reduction in fracture risk with a $1 \%$ to $6 \%$ improvement in spine BMD [39]. While the study was not designed specifically to measure the effectiveness of treatment, we observed that osteoporotic women who initiated an osteoporosis treatment during follow-up had a lower 10-year cumulative incidence of fracture than did the non-treated women. This difference could either be reflective of therapy or the type of patients who started therapy. While similar trends indicating the correlation between baseline BMD and fracture risk were observed in the analyses stratified by fracture subtypes, the incidence of hip fracture did not differ between normal and osteopenia group among the treated subgroup. These results may have been affected by the time-varying effect of external risk factors for fracture during the follow-up, or, alternatively, it may reflect the limitation of using spine BMD to predict the risk of hip fracture.

The population-based screening program in South Korea has a valuable predictive role-identifying subjects with low bone mass and providing an index for the prediction of long-term fracture risk. In these national BMD screening data, 58.4\% of women who developed fragility fractures had baseline BMD Tscores $>-2.5$. While the magnitude of fracture risk was substantial among women in the osteopenia range, these patients were not necessarily recommended pharmacologic interventions unless they had a history of fracture, $\geq 20 \%$ fracture risk assessment tool 10-year probability of major fragility fracture, or $\geq 3 \% 10$-year probability of hip fracture [16,40,41]. A recent update in the South Korean health insurance plan extended the reimbursement coverage of bisphosphonates, SERM, and active vitamin D3 to those who had recent fragility fractures or women with osteopenia who had $>90$ days of exposure to glucocorticoids within the past 6 months [42]. However, the majority of women with osteopenia without such conditions are not indicated for these treatments according to the current reimbursement criteria. Subsequently, the BMD rescreening rate was not sufficiently high compared with the recommendation of the United States Preventive Services Task Force, suggesting BMD screening for women aged $\geq 65$ years [43]. Therefore, there is an unmet public health need for targeted efforts toward the early identification and treatment of women with osteopenic BMD who have a high risk of fracture.

Our study has several strengths. Our study provides long-term epidemiological evidence indicating that the magnitude of fracture risk in the Asian population is substantial. To our knowledge, this is the largest population based study to examine postmenopausal fragility fracture risk in an Asian population. Due to our use of a nationwide healthcare claims database, all subjects' reimbursed healthcare encounters (including diagnostic records, 
prescriptions, or procedures) were retrieved, and therefore, artificial follow-up loss due to a transfer to a different health care provider or death is unlikely to have occurred in our study. In contrast, artificial follow-up loss is a frequent source of outcome misclassification bias in previous studies using survey data or electronic health records, which may lead to under-detection of study outcomes. The artificial follow-up loss may also give rise to selection bias, given that patients with an incomplete followup due to transfer to advance healthcare centers (e.g., long-term nursing facilities) are likely to have differential clinical characteristics to those who remained in the study cohort. Furthermore, our findings have solid external validity because of the assembly of data from a nationwide screening program in which the study subjects were followed longitudinally.

However, our study has several limitations. First, this study has a potential limitation of misclassification of BMD group. As we used lumbar spine BMD values from national health screening, osteoporotic patients with low hip BMD may have been included in the osteopenia group. It is possible that the study subjects with osteoporotic cortical BMD, but with osteopenic trabecular BMD, were defined as osteopenia group. With the use of spine BMD testing results, the risk for vertebral fractures could differ the most, and these results also show the predictive role of spine BMD for hip or NHNV fractures. The plausible reason could be that trabecular BMD may serve as a proxy marker for the overall decrease in BMD because trabecular bone responds to metabolic changes more sensitively than does cortical bone [44]. Second, the data were primarily collected for health insurance claims for reimbursement purposes; thus, the inherent limitation of health insurance claims data may exist in the form of incomplete, inaccurate, or missing data [45]. The prevalence of baseline clinical characteristics may have been affected by the potential miscoding of diagnostic codes. A validation study comparing the diagnosis records of the South Korean healthcare database with electronic medical records indicated an overall positive predictive value of $82 \%$ [16]. Third, only a onetime BMD measurement at the age of 66 years was available, and we could not observe BMD changes during the follow-up period. Fourth, it is unclear whether post-diagnostic procedures, such as patient counseling and education [46], were implemented in subjects with osteopenia or osteoporosis, and the consequences of these interventions were not quantified. Information on medication intake for patients with osteopenia was unavailable in this database, as it is not reimbursed by the NHIS in Korea. Fifth, we cannot exclude the possibility that results from BMD screening and clinical diagnosis of osteoporosis and os- teopenia can be different, although BMD screening is considered the most commonly used indicator. Finally, not all relevant data are available within the data source. Of 10 risk factors for fracture that are commonly considered in clinical practice [47], the data source for this study had data on eight risk factors (covariates of age, sex, systemic glucocorticoid use, rheumatoid arthritis diagnosis, prior fracture, BMI, alcohol use, smoking status), but did not include data on the other two risk factors (femoral neck BMD, and parental history of fracture).

In conclusion, the long-term fracture risk in postmenopausal women is substantial in patients with osteopenia and osteoporosis. Despite advances in diagnosis and preventive treatments implemented in recent years, recommendations and guidelines for post-BMD screening still need to be improved. In addition, it is important to note that over half of the fractures within 10 years occurred in women with modestly reduced BMD, including women with osteopenia, which warrants targeted efforts toward early identification and implementation of preventive strategies for women who are in the osteopenia range but at a high risk of fracture. This study supports the notion that public health policy should aim to reduce the burden of fragility fractures.

\section{CONFLICTS OF INTEREST}

This study was sponsored by Amgen, Inc. This work was supported by a grant (21153MFDS607) from Ministry of Food and Drug Safety of South Korea in 2021-2025. Medical writing and editorial assistance were provided by Mami Hirano, MS, of Cactus Life Sciences (part of Cactus Communications), and funded by Amgen, Inc.

Ju-Young Shin received grants from pharmaceutical companies, including Amgen, Pfizer, Hoffmann-La Roche, Dong-A ST, and Yungjin, outside the submitted work. Yunji Hwang and Jeffrey L. Lange are employees and stockholders of Amgen, Inc. Yeon-Hee Baek is an employee of Amgen Korea. Sun Wook Cho, Han Eol Jeong, and Ju Hwan Kim have no conflicts of interest to declare.

\section{ACKNOWLEDGMENTS}

Ju-Young Shin received grants from the Ministry of Food and Drug Safety, the Ministry of Health and Welfare, the National Research Foundation of Korea, and a government-wide R\&D Fund for Infectious Disease Research.

The authors sincerely thank Hyuna Lim and the medical team (Amgen Korea) for helping with the study management, Cae 
Tolman (Amgen Asia) for providing medical insights and review comments, and Pf. Sung Hye Kong, M.D. (Seoul National University Bundang Hospital) for providing medical advice.

\section{AUTHOR CONTRIBUTIONS}

Conception or design: Y.H.B., S.W.C., Y.H., J.L.L., J.Y.S. Acquisition, analysis, or interpretation of data: Y.H.B., S.W.C., H.E.J., J.H.K., Y.H., J.L.L., J.Y.S. Drafting the work or revising: Y.H.B., S.W.C., H.E.J., J.H.K., Y.H., J.L.L., J.Y.S. Final approval of the manuscript: Y.H.B., S.W.C., H.E.J., J.H.K., Y.H., J.L.L., J.Y.S.

\section{ORCID}

Yeon-Hee Baek https://orcid.org/0000-0001-9597-6310

Sun Wook Cho https://orcid.org/0000-0002-7394-3830

Ju-Young Shin https://orcid.org/0000-0003-1010-7525

\section{REFERENCES}

1. Lin JT, Lane JM. Osteoporosis: a review. Clin Orthop Relat Res 2004;425:126-34.

2. Ji MX, Yu Q. Primary osteoporosis in postmenopausal women. Chronic Dis Transl Med 2015;1:9-13.

3. Schott AM, Cormier C, Hans D, Favier F, Hausherr E, Dargent-Molina $\mathrm{P}$, et al. How hip and whole-body bone mineral density predict hip fracture in elderly women: the EPIDOS Prospective Study. Osteoporos Int 1998;8:247-54.

4. The relationship between bone density and incident vertebral fracture in men and women. J Bone Miner Res 2002;17: 2214-21.

5. Van der Klift M, De Laet CE, McCloskey EV, Hofman A, Pols HA. The incidence of vertebral fractures in men and women: the Rotterdam Study. J Bone Miner Res 2002;17: 1051-6.

6. Siris ES, Chen YT, Abbott TA, Barrett-Connor E, Miller PD, Wehren LE, et al. Bone mineral density thresholds for pharmacological intervention to prevent fractures. Arch Intern Med 2004;164:1108-12.

7. Lo SS. Bone health status of postmenopausal Chinese women. Hong Kong Med J 2015;21:536-41.

8. Tian L, Yang R, Wei L, Liu J, Yang Y, Shao F, et al. Prevalence of osteoporosis and related lifestyle and metabolic factors of postmenopausal women and elderly men: a crosssectional study in Gansu province, Northwestern of China.
Medicine (Baltimore) 2017;96:e8294.

9. Park EJ, Joo IW, Jang MJ, Kim YT, Oh K, Oh HJ. Prevalence of osteoporosis in the Korean population based on Korea National Health and Nutrition Examination Survey (KNHANES), 2008-2011. Yonsei Med J 2014;55:1049-57.

10. Barrett-Connor E, Siris ES, Wehren LE, Miller PD, Abbott TA, Berger ML, et al. Osteoporosis and fracture risk in women of different ethnic groups. J Bone Miner Res 2005;20: 185-94.

11. Kung AW, Lee KK, Ho AY, Tang G, Luk KD. Ten-year risk of osteoporotic fractures in postmenopausal Chinese women according to clinical risk factors and BMD T-scores: a prospective study. J Bone Miner Res 2007;22:1080-7.

12. Seong SC, Kim YY, Park SK, Khang YH, Kim HC, Park $\mathrm{JH}$, et al. Cohort profile: the National Health Insurance Service-National Health Screening Cohort (NHIS-HEALS) in Korea. BMJ Open 2017;7:e016640.

13. Kim HS, Shin DW, Lee WC, Kim YT, Cho B. National screening program for transitional ages in Korea: a new screening for strengthening primary prevention and followup care. J Korean Med Sci 2012;27(Suppl):S70-5.

14. National Health Insurance Service. National Health Screening Statistical Yearbook. Seoul: NHIS; 2016.

15. Seong SC, Kim YY, Khang YH, Park JH, Kang HJ, Lee H, et al. Data resource profile: the National Health Information Database of the National Health Insurance Service in South Korea. Int J Epidemiol 2017;46:799-800.

16. Park EC, Jang SI, Jeon SY, Lee SA, Lee JE, Choi DW. Report of the evaluation for validity of discharged diagnoses in Korean Health Insurance database. Seoul: Health Insurance Review and Assessment Service; 2017.

17. Blake GM, Fogelman I. Peripheral or central densitometry: does it matter which technique we use? J Clin Densitom 2001;4:83-96.

18. Ministry of Health and Welfare of Korea. Criteria for Health Examination [Internet]. Sejong: Korean Law Information Center; 2014 [cited 2021 Oct 19]. Available from: https:// www.law.go.kr/LSW/admRulInfoP.do?admRulSeq $=20000$ 00118324\#AJAX.

19. World Health Organization. Prevention and management of osteoporosis: report of a WHO scientific group. Geneva: World Health Organization; 2003.

20. Bultink IE, Baden M, Lems WF. Glucocorticoid-induced osteoporosis: an update on current pharmacotherapy and future directions. Expert Opin Pharmacother 2013;14:185-97.

21. Lee EA, Shin DW, Yoo JH, Ko HY, Jeong SM. Anemia and 
risk of fractures in older Korean adults: a nationwide population-based study. J Bone Miner Res 2019;34:1049-57.

22. Ross PD. Risk factors for osteoporotic fracture. Endocrinol Metab Clin North Am 1998;27:289-301.

23. Weisell RC. Body mass index as an indicator of obesity. Asia Pac J Clin Nutr 2002;11:S681-4.

24. Blum MR, Bauer DC, Collet TH, Fink HA, Cappola AR, da Costa BR, et al. Subclinical thyroid dysfunction and fracture risk: a meta-analysis. JAMA 2015;313:2055-65.

25. Janghorbani M, Van Dam RM, Willett WC, Hu FB. Systematic review of type 1 and type 2 diabetes mellitus and risk of fracture. Am J Epidemiol 2007;166:495-505.

26. Li C, Zeng Y, Tao L, Liu S, Ni Z, Huang Q, et al. Meta-analysis of hypertension and osteoporotic fracture risk in women and men. Osteoporos Int 2017;28:2309-18.

27. Smith BJ, Phillips PJ, Heller RF. Asthma and chronic obstructive airway diseases are associated with osteoporosis and fractures: a literature review. Respirology 1999;4:101-9.

28. Akyea RK, McKeever TM, Gibson J, Scullion JE, Bolton CE. Predicting fracture risk in patients with chronic obstructive pulmonary disease: a UK-based population-based cohort study. BMJ Open 2019;9:e024951.

29. Xue AL, Wu SY, Jiang L, Feng AM, Guo HF, Zhao P. Bone fracture risk in patients with rheumatoid arthritis: a metaanalysis. Medicine (Baltimore) 2017;96:e6983.

30. Pasco JA, Seeman E, Henry MJ, Merriman EN, Nicholson GC, Kotowicz MA. The population burden of fractures originates in women with osteopenia, not osteoporosis. Osteoporos Int 2006;17:1404-9.

31. Wright NC, Looker AC, Saag KG, Curtis JR, Delzell ES, Randall S, et al. The recent prevalence of osteoporosis and low bone mass in the United States based on bone mineral density at the femoral neck or lumbar spine. J Bone Miner Res 2014;29:2520-6.

32. Bagger YZ, Tanko LB, Alexandersen P, Hansen HB, Qin G, Christiansen $\mathrm{C}$. The long-term predictive value of bone mineral density measurements for fracture risk is independent of the site of measurement and the age at diagnosis: results from the Prospective Epidemiological Risk Factors study. Osteoporos Int 2006;17:471-7.

33. Kung AW, Luk KD, Chu LW, Tang GW. Quantitative ultrasound and symptomatic vertebral fracture risk in Chinese women. Osteoporos Int 1999;10:456-61.

34. Fujiwara S, Kasagi F, Masunari N, Naito K, Suzuki G, Fukunaga $M$. Fracture prediction from bone mineral density in Japanese men and women. J Bone Miner Res 2003;18:1547-53.
35. Xia WB, He SL, Xu L, Liu AM, Jiang Y, Li M, et al. Rapidly increasing rates of hip fracture in Beijing, China. J Bone Miner Res 2012;27:125-9.

36. Bow CH, Cheung E, Cheung CL, Xiao SM, Loong C, Soong $\mathrm{C}$, et al. Ethnic difference of clinical vertebral fracture risk. Osteoporos Int 2012;23:879-85.

37. Chie WC, Yang RS, Liu JP, Tsai KS. High incidence rate of hip fracture in Taiwan: estimated from a nationwide health insurance database. Osteoporos Int 2004;15:998-1002.

38. Cheung CL, Ang SB, Chadha M, Chow ES, Chung YS, Hew FL, et al. An updated hip fracture projection in Asia: the Asian Federation of Osteoporosis Societies study. Osteoporos Sarcopenia 2018;4:16-21.

39. Cummings SR. How drugs decrease fracture risk: lessons from trials. J Musculoskelet Neuronal Interact 2002;2:198200.

40. Cosman F, de Beur SJ, LeBoff MS, Lewiecki EM, Tanner B, Randall S, et al. Clinician's guide to prevention and treatment of osteoporosis. Osteoporos Int 2014;25:2359-81.

41. Camacho PM, Petak SM, Binkley N, Clarke BL, Harris ST, Hurley DL, et al. American Association of Clinical Endocrinologists and American College of Endocrinology clinical practice guidelines for the diagnosis and treatment of postmenopausal osteoporosis: 2016. Endocr Pract 2016;22(Suppl 4):1-42.

42. Health Insurance Review \& Assessment Service. Seoul Branch: a guide for reimbursement of health insurance claims [Internet]. Wonju: HIRA; 2021 [cited 2021 Oct 19]. Available from: https://www.hira.or.kr/sViewer/index.do?ebookSn=635.

43. US Preventive Services Task Force, Curry SJ, Krist AH, Owens DK, Barry MJ, Caughey AB, et al. Screening for osteoporosis to prevent fractures: US Preventive Services Task Force recommendation statement. JAMA 2018;319:2521-31.

44. Frost HM. Cybernetic aspects of bone modeling and remodeling, with special reference to osteoporosis and whole-bone strength. Am J Hum Biol 2001;13:235-48.

45. Stein JD, Lum F, Lee PP, Rich WL 3rd, Coleman AL. Use of health care claims data to study patients with ophthalmologic conditions. Ophthalmology 2014;121:1134-41.

46. Shin HY, Kang HC, Lee K, Park SM. Association between the awareness of osteoporosis and the quality of care for bone health among Korean women with osteoporosis. BMC Musculoskelet Disord 2014;15:334.

47. Kanis JA, Johnell O, Oden A, Johansson H, McCloskey E. FRAX and the assessment of fracture probability in men and women from the UK. Osteoporos Int 2008;19:385-97. 